\section{CORRELATIVE ANALYSIS OF BLOOD AND BIOPSY SAMPLES FROM A CLINICAL TRIAL OF HSP9O INHIBITION IN COMBINATION WITH PEMBROLIZUMAB REVEALS INCREASED INTRATUMORAL MYELOID CELL ACCUMULATION AFTER TREATMENT}

${ }^{1}$ Cameron Herting* ${ }^{*}{ }^{2}$ Yuchen Zhang, ${ }^{1}$ Deon Doxie, ${ }^{1}$ Michael Ware, ${ }^{1}$ Olatunii Alese ${ }^{1}$ Christina Wu, ${ }^{1}$ Mehmet Akce, ${ }^{1}$ Mohammad Zaidi, ${ }^{1}$ Amanda Ruggieri, ${ }^{1}$ Madhav Dhodapkar, 'Kavita Dhodapkar, 'Juan Sarmiento, ${ }^{1}$ Rafi Ahmed, 'Shishir Maithel, ${ }^{1}$ Bassel El-Rayes, ${ }^{1}$ Gregory Lesinski. ${ }^{1}$ Emory University, Decatur, GA, USA; ${ }^{2}$ Xi'an Jiaotong University, Shaanxi, China

Background Pancreatic ductal adenocarcinoma (PDAC) has yet to widely benefit from $\mathrm{T}$ cell-targeted immunotherapy and displays universally poor prognosis. Thus, enhancing the activity of immunotherapy is a high priority. Our laboratory recently reported that heat shock protein-90 (Hsp90) inhibition enhances the efficacy of PD-1 blockade in murine models of PDAC (Zhang Y. et al., Mol Cancer Ther, 2020). Hsp90 inhibitors can limit activation of cancer associated fibroblasts (CAF) and promote infiltration of $\mathrm{T}$ cells when combined with $\mathrm{PD}-1$ blockade in preclinical systems.

Methods Based on these data, we are conducting a Phase Ib/II clinical trial to evaluate the combination of XL888 (Hsp90 inhibitor) and pembrolizumab in patients with metastatic pancreatic cancer. We hypothesize that this combination will be safe and elicit pronounced microenvironmental changes, leading to enhanced efficacy of checkpoint blockade in a tumor type that is otherwise refractory to this approach. During the phase II portion patients were randomized to receive a three week lead in with either pembrolizumab or pembrolizumab and XL888. Paired biopsies and blood samples were obtained at baseline and at week two on treatment and CyTOF was used to assess changes in circulating and tumor infiltrating immune populations. Further, CyTOF profiling of circulating immune cells was performed to assess impacts of XL888 on over thirty phenotypically defined immune populations (figure 1).

Results As of June 2021, paired liver biopsy specimens from sites of metastasis have been successfully obtained from a total of 8 patients and paired peripheral blood mononuclear cell samples have been analyzed in 24 patients. Our CyTOF analysis illustrated a surprising increase in myeloid cell populations within the tumor following treatment. Analysis of circulating immune cells illustrated a decrease in natural killer cells and Th17 populations following treatment while naïve $B$ cells were increased. These data will be validated by immunohistochemical analysis of FFPE biopsy specimens obtained in parallel at the time of CyTOF analysis. The impact of XL888 on systemic cytokines and chemokines $(n=48$ total) in the peripheral blood from patients enrolled in the clinical trial is therefore being assessed as a potential mechanism to explain this observation.

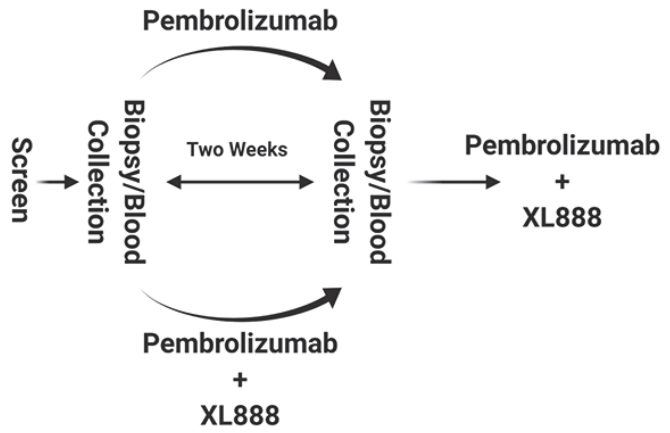

Plasma

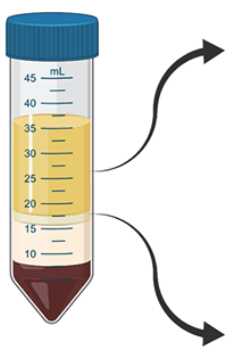

Multiplex analysis of circulating chemokines, cytokines, and growth factors

PBMC

Circulating immune cell profiling with Maxpar Direct Immune Profiling Assay

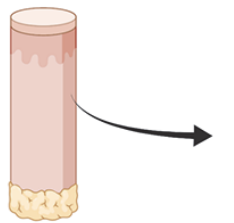

Biopsy

Dissociation and profiling of tumor infiltrating immune cells with CyTOF

Abstract 403 Figure 1 Clinical trial and correlative analysis schema. Patients were randomized to receive either pembrolizumab alone or in combination with the HSP90 inhibitor XL888 for a two week cycle prior to crossover to the combination arm. Plasma, peripheral blood mononuclear cells (PBMC), and biopsies were assayed to evaluate immunomodulatory effects of the therapies.

Conclusions Clinical data from this trial indicates that this combination is safe in patients. As clinical data matures, changes in soluble and cellular biomarkers will be correlated with response to elucidate mechanisms of response or resistance to this combination therapy.

Trial Registration This clinical trial is underway and registered with the ID NCT03095781

Ethics Approval The study was approved by Emory University's Ethics Board, approval IRB00087397.

Consent Written informed consent was obtained from the patient for publication of this abstract and any accompanying images. A copy of the written consent is available for review by the Editor of this journal.

http://dx.doi.org/10.1136/jitc-2021-SITC2021.403 